\title{
Patient Safety in Laboratory Medicine
}

\author{
Mario Plebani, Ada Aita, and Laura Sciacovelli
}

\section{Learning Objectives}

The notion of patient safety in laboratory medicine can be loosely interpreted as the assurance that harm to patients is prevented, safe care outcomes are enhanced through error prevention, and the system is continuously improved. However, this somewhat simplistic interpretation hides the concept's true complexity. A clear definition of the laboratory professional's role and competencies in the diagnostic process is therefore required, as is the use of suitable quality assurance tools.

The concept of a "brain-to-brain loop" of laboratory testing encompasses the different steps involved, starting with appropriate test requesting and concluding with the appropriate use of laboratory information (Fig. 24.1). Moreover, it highlights the importance of integrating laboratory information in the care pathways, calling for system quality and patient safety, and also elucidates the value of laboratory medicine.

M. Plebani $(\bowtie) \cdot$ A. Aita

Department of Laboratory Medicine, UniversityHospital of Padova, Padova, Italy

Department of Medicine-DIMED, University of Padova, Padova, Italy

e-mail: mario.plebani@unipd.it

L. Sciacovelli

Department of Laboratory Medicine, UniversityHospital of Padova, Padova, Italy

e-mail: laura.sciacovelli@aopd.veneto.it
In the last few decades, changes in the nature of laboratory services have underscored a paradigmatic transformation of the laboratory scenario [1] thanks to technological innovation, the introduction of ever more complex tests in emerging diagnostic fields, more advanced diagnostics, and other internal and external drivers. The transformation will become even more marked in the future. In the ten points identified in the "Manifesto for the future of Laboratory Medicine professionals" (Table 24.1), great emphasis is placed on the importance of cooperation in reducing the risk of diagnostic errors, the implementation of reliable laboratory medicine stewardship, the involvement of laboratory medicine professionals in interdisciplinary teams, and the promotion of professional expertise. A large body of evidence demonstrates that this integration is crucial to the rational utilization of laboratory information [2].

The issue of patient safety, which impacts every diagnosis, involves numerous stakeholders. No single intervention to prevent errors is available, and there is a pressing need for the rigorous evaluation of possible solutions that will be beneficial, will obviate unintended consequences, and, above all, will safeguard patients.

In the healthcare setting, medical laboratories should always be a driver in ensuring patient safety through:

- The integration of laboratory professionals with new competencies and skills in 
Fig. 24.1 The brain-to-brain-loop: description of the total testing process (from reference 8 modified)

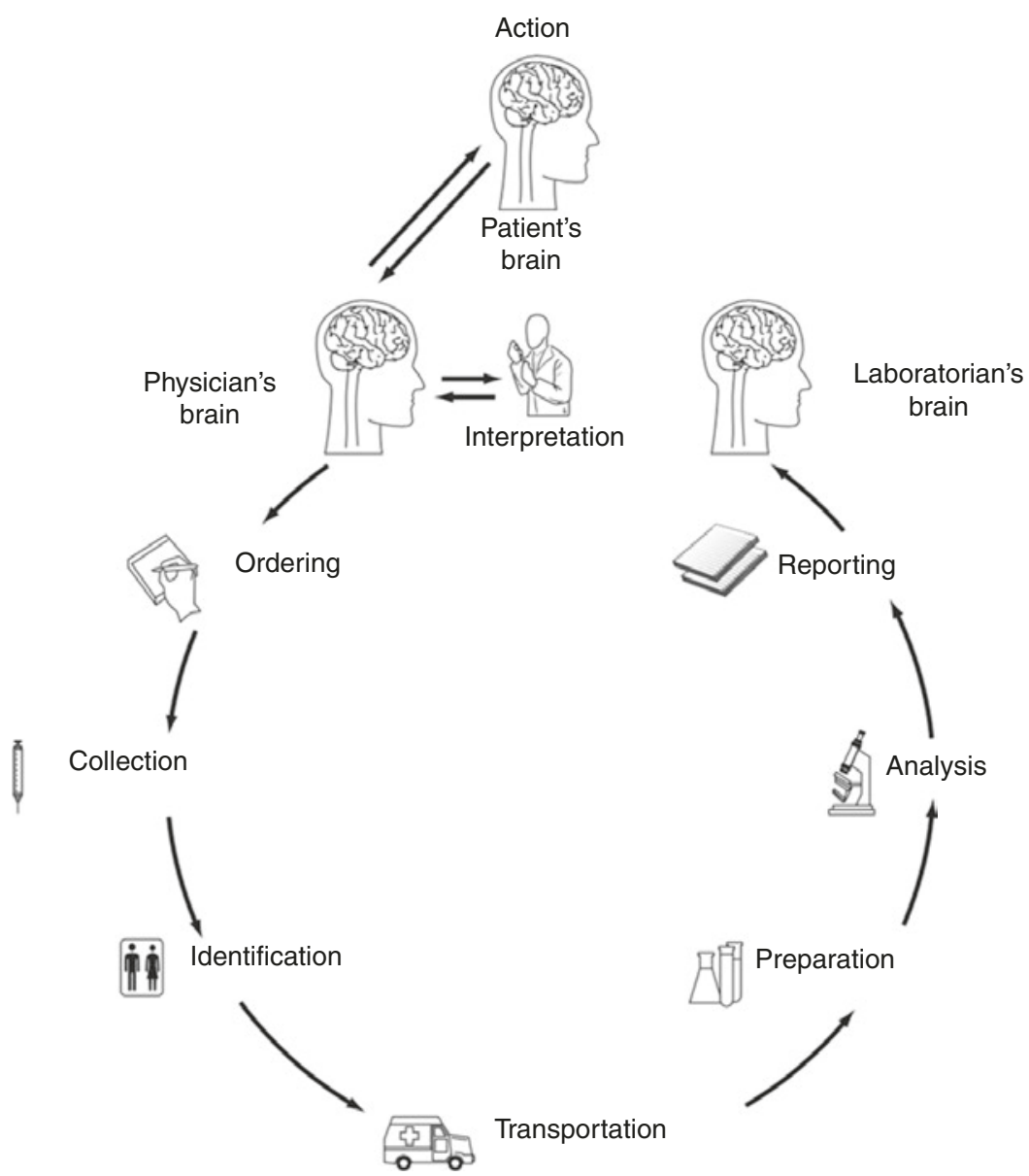

Table 24.1 Manifesto for the future of laboratory medicine professionals [1]

1. Convert results in clinical information

2. Cooperate in reducing the risk of diagnostic errors

3. Implement a reliable laboratory medicine stewardship

4. Combine data of all laboratories subspecialties and diagnostic imaging in the same report

5. Establish reliable reference ranges and decision limits

6. Facilitate more effective teamwork and be actively involved in interdisciplinary teams

7. Promote the shift from volume based reimbursement models to clinical values

8. Improve and update the way laboratory medicine is taught

9. Do not neglect administrative competence and duties

10. Promote the value of the profession multidisciplinary teams, in which all stakeholders are involved.

- Awareness of the impact of laboratory errors in patient management.

- The implementation of effective quality assurance tools to identify and prevent potential laboratory errors.

\subsection{Epidemiology of Adverse Events}

\subsubsection{Laboratory Medicine as a Driver in Ensuring Patient Safety}

The phrase "clinical laboratory stewardship" effectively clarifies the indispensable role of 
laboratory medicine in healthcare and points to the need to promote a new vision for the discipline in which some novel ideas must be stressed and focused on. Diagnostic and therapeutic networks must be promoted in order to improve patient-centered and end-to-end support in clinical pathways and to transform laboratory data into effective information. Studies in literature highlight the urgent need for clinicians to make better use of diagnostic testing and to be confident that laboratory information issued to them is accurate. Important issues must be shared in order to achieve compliance with the above needs. In order to reduce errors and improve quality of care, laboratory professionals must be made aware of the impact laboratory results have on the patient and, on the other hand, clinicians must have a thorough understanding of the tools implemented and used in the laboratory [3-5].

In the healthcare system, Laboratory Medicine is still considered a low-risk speciality with respect to other specialities such as emergency and intensive care medicine [6]. This is because the main activities in laboratory medicine are accurately defined $[7,8]$ and are more easily controlled than procedures in an emergency department, where they are strictly dependent on the healthcare professionals. However, laboratory errors tend to be more insidious and difficult to immediately identify because they involve several steps, numerous providers, and a greater time lapse between testing, physician action, and patient outcome [9].

During the healthcare process, failures occurring in a phase nearest to patient intervention are more likely to result in patient harm due to the presence of active and defensive barriers designed to identify the event before it impacts patient outcome. However, despite the common belief that errors in laboratory testing have less of an impact on patient safety than those occurring in the operating or resuscitation rooms, several examples in literature demonstrate that errors in the testing process can result in a negative patient outcome. In a survey, anatomic pathologists and medical laboratory directors were asked questions on error rates, on barriers to error disclosure, and on their experience with pathology and laboratory error disclosure. Of the $95.2 \%$ of respondents who reported having experienced an error, $43.6 \%$ reported involvement with a serious error, $69.1 \%$ a minor error, and $77.6 \%$ a near miss [10]. Himmel et al. described a case of unnecessary hemodialysis being performed in a healthy patient due to an erroneously transcribed methanol result $(6 \mathrm{mmol} / \mathrm{L}$ instead of $0.06 \mathrm{mmol} / \mathrm{L})$ [11]. In 2007 in Italy, a transcription error in an HIV result led to the death of three transplant recipients [12]. Adverse events have been also associated with false glucose readings when using glucose dehydrogenase pyrroloquinoline quinone (GPD-PQQ) test strips: out of 82 erroneous reports, 16 (20\%) led to death, $46(56 \%)$ severe hypo-glycemia, and 12 (15\%) minor hypo-glycemia [13]. Other authors have reported that about $30 \%$ of cases of error in total laboratory testing translate into a patient care problem (e.g., inappropriate admission to critical care units, inappropriate transfusions, modifications in heparin and digoxin therapies) [14-16].

These data demonstrate the importance of laboratory information in clinical decisionmaking since it can significantly affect the diagnosis and subsequent patient management [17]. The reliability of laboratory information is therefore the prerequisite for assuring a quality healthcare process and reducing the risk of harm to patients.

Although it is acknowledged worldwide that the fundamental role of laboratory medicine is to ensure patient safety, more could be done to highlight this role in order to make the laboratory's work visible to the patient.

\subsubsection{From Laboratory-Related Errors to Diagnostic Errors}

The understanding of errors in terms of type, frequency, causes, and impact on patients is crucial to identifying and implementing control measures to prevent failures and reduce risk. In the laboratory field, the meaning of "error" has changed over time, in parallel with the transformation of the organizational methods of the med- 
ical laboratory and the definition of the laboratory testing process itself.

In the late 1990s, the promotion of patientcentered care prompted laboratory professionals to investigate any defects in the TTP that could negatively impact the patient. A series of papers published between 1989 and 2007 documented a high error rate in the pre- and post-analytical steps, thus demonstrating the high vulnerability of these phases [15, 16, 18, 19]. Accordingly, the accepted definition of laboratory error became "a defect occurring at any part of the laboratory cycle, from ordering tests to reporting results and appropriately interpreting and reacting on these," which was also incorporated into the ISO Technical Report 22367:2008 "Medical laboratoriesReduction of error through risk management and continual improvement-Complementary elements" [20].

In 2011, the Institute of Medicine (IOM) included the service of laboratory medicine among the ten categories of essential services in the United States health system, marking a new era for the medical laboratory. As the medical laboratory becomes an integral part of the healthcare system, laboratory-related error becomes part of a much wider issue, known as "diagnostic error" [21, 22]. Diagnostic errors have been defined as failures to provide an accurate and timely explanation of the patient's health problems and/or communicate that explanation to the patient. Diagnostic errors are typically subdivided into errors in which diagnosis has been (a) delayed, despite sufficient available information, (b) wrong, because a diagnosis was made before the correct one was determined, or (c) missed, when no diagnosis was made [23]. The concept of "diagnostic errors" definitively links laboratory-associated errors to patient safety problems. The fundamental role of the medical laboratory in the diagnostic process was highlighted by a survey administered to clinicians to assess type and causes of missed and delayed diagnoses. The results showed that errors occurred most frequently in the testing phase (i.e., failure to order, report, and follow-up laboratory results) (44\%), followed by errors in clinician assessment (overruling or failing to consider a competing diagnosis) (32\%), history taking
(10\%), physical examination (10\%), and referral or consultation errors and delays (3\%) [24].

Failure to order appropriate diagnostic tests, including laboratory tests, has also been linked to missed and delayed diagnoses in the ambulatory setting (55\%) [25], and in emergency departments (58\%). Likewise, failure to note abnormal test results (7\%) has been related to delay in diagnosing cancer. The incorrect interpretation of laboratory tests, resulting in diagnostic errors, has also been documented in the primary care setting (37\%), internal medicine (38\%), and emergency departments $(37 \%)[25,26]$.

Current research into laboratory-related diagnostic errors highlights the following.

- Pre-and post-analytical phases are more vulnerable to errors, accounting for 46-68\% and $19-47 \%$ of errors, respectively. The management of the interface between the clinical setting and laboratory remains a challenge for healthcare professionals [27].

- Analytical quality is a persistent issue. Initial studies on laboratory-related errors focused on the analytical phase, the only phase completely performed within the laboratory walls and under direct laboratory control. Furthermore, only errors in the measurement of clinical chemistry analytes were considered. Despite their limited design, these studies offered a wide range of strategies to improve analytical performance and provide clinicians with timely and reliable results. The strategies adopted (e.g., the development of external quality assurance (EQA) programs, the improvement of internal quality control (IQC), regulation and standardization of analytical techniques and reagents, automation and computerization of laboratory processes) led to a dramatic decrease in analytical error rates and to a significant increase in test demand and utilization.

- Analytical interferences must be focused on. Despite the optimism of clinical pathologists, analytical interferences still affect many tests, such as glucose, bilirubin, C-reactive protein, creatinine, and albumin [28]. These errors, also known as irregular errors, represent one of the greatest challenges for laboratory pro- 
fessionals because they are not detectable by quality control procedures, are reproducible within the test system, and may be clinically plausible. Furthermore, their frequency is variable and probably underreported [29]. Immunoassays are the most affected tests, with an analytical error rate of $0.4-4 \%$, considerably higher than those for other routine tests and in some cases associated with an adverse clinical outcome (e.g., unnecessary hysterectomy and chemotherapy in a 22 -yearold woman due to false-positive hCG results caused by heterophilic antibodies) [30]. The issue of irregular analytical errors presents an opportunity to reframe the mission of laboratory medicine as the provision of key information for effective clinical decision-making and optimal patient outcome [31].

- Errors do not only concern clinical chemistry tests. New pathophysiological knowledge and technologies have led to the introduction of novel, ever more sophisticated tests into clinical practice, calling for further efforts to ensure competency of laboratory staff as well as other healthcare professionals [1]. Although the test cycle is the same across laboratory medicine tests and disciplines, this cannot be said for the distribution of the errors within the test cycle. In molecular-genetic testing or mass spectrometry, many steps of the analytical process are not yet automated and are closely linked to the inherent judgment of the laboratory professional, thus making the results more subjective than those of other clinical laboratory tests. In molecular-genetic testing for example, it has been demonstrated that $60 \%$ of errors occur in the pre-analytical phase, $32 \%$ in the analytical phase, and $8 \%$ in the post-analytical phase [32].

\subsection{Safety Practices and Implementation Strategy}

In recent years, the evolution of the role of laboratory medicine in patient management and the growing attention to cost containment have underscored an evaluation of the service provided by the medical laboratory based on efficacy criteria. In this context, improvement has been observed in quality control techniques, from improvements in the analytical phase to the promotion and development of quality assurance systems for the entire analysis process; several quality improvement initiatives have been undertaken to support sustainable outcomes based on systematic and organizational criteria.

A body of scientific evidence shows that it is now essential to identify opportunities for improvement while considering all TTP activities, especially those in the pre- and postanalytical phases. Systems for the identification and monitoring of errors are important quality assurance tools, as are proactive and reactive analysis methods that focus not only on the processes themselves but also, and above all, on any risk to the patient.

Of the procedures to be implemented in medical laboratories, the identification and monitoring of errors is paramount to ensure proper performance because it calls for continuous data analysis and the implementation of preventive, corrective, and ameliorative actions whenever necessary. The monitoring of result accuracy, a traditional laboratory process, is conducted using IQC procedures and through participation in EQA programs. In the last few decades, the responsibility of laboratory professionals has widened thanks to awareness of the brain-tobrain loop (Fig. 24.1) [33]. The systematic use of approved quality indicators (QIs) to control critical TTP activities over time has expanded to the monitoring of the extra-analytical phases and the description of the efficiency and effectiveness of performance.

Moreover, a robust, well-structured, and wellmanaged quality management system can provide a wide variety of information generated by both symptomatic events (e.g., incident reporting) and asymptomatic events (e.g., analysis of strengths, weaknesses, opportunities, and threats) for measuring and monitoring different aspects of the process and its outcomes.

Examples of information sources are:

- Reports on participation in EQA programs.

- Quality indicator data. 
- Findings of external accreditation and/or certification audits.

- Records of non-conformities (e.g., errors, complaints, adverse events, non-compliance).

- Findings of surveys investigating user satisfaction, where the user is understood to be one who utilizes the service (e.g., citizen, patient, clinician, doctor) or one who works within the process as a user of one sub-process and a supplier of another.

- Assessments of staff competency.

The analysis of all data collected contributes to the definition of organizational and quality objectives and of intervention priorities. However, the effectiveness of the information for improvement purposes is influenced by the criteria and methods with which the information is collected and subsequently managed.

Only a small proportion of laboratory errors give rise to actual patient harm and adverse events, thanks to several checks and defensive layers implemented to guarantee the reliable release of laboratory information; however, each and every error that does occur must serve as an important learning opportunity [34].

\subsubsection{ISO 15189 Accreditation}

The reliability of laboratory tests has increased dramatically in line with technological progress and the refinement of techniques, methods, and professional skills. For a medical laboratory to reliably deliver routine services at high volumes continuously and broadly, it must emphasize the importance of drawing maximum attention to the quality of its processes, starting with the biological sample quality (i.e., collection, handling, and transport), followed by analytical accuracy, and then the quality of report communication (e.g., timeliness, appropriateness of reference intervals/decision levels, controls in place to ensure the correct communication is received by clinicians).

Although a robust quality management system is crucial for the correct management and traceability of all processes, it is now more important than ever to understand how to optimize efficiency and effectiveness while overcoming the mentality of blind compliance with requirements. The definition and application of criteria, procedures, and quality assurance tools must be driven by laboratory professionals on the basis of practice and data analysis, rather than by requirements defined outside the laboratory.

Improvement action plans for laboratory activities are multiple and varied due to the complexity of the relationships and interactions between the different processes and activities. Success depends on:

- The commitment of leadership to improving quality as a modus operandi.

- An organization-wide culture that recognizes the need for and calls for the involvement of all personnel in improvement activities.

- Integrated and defined processes and procedures describing the ways in which improvement can be implemented and responsibilities articulated.

- The application by management and all staff members of knowledge and skills relevant to the continuous improvement of concepts, models, and tools [35].

Laboratory medicine is increasingly recognized as a fundamental component of patient care and therefore laboratory professionals are requested to improve not only analytical but also clinical competence. Great efforts have been made by laboratory professionals to supplant the belief that the recognition of performance quality might be assured with the granting of ISO 9001 certification, highlighting instead the suitability of the ISO 15189 accreditation process.

Accreditation in compliance with ISO 15189 (Medical laboratories-Requirements for quality and competence) is designed to demonstrate the reliability of laboratory performances to patients and users, and general stakeholders [36]. This assurance is based on the implementation of an adequate quality management system and, above all, on the availability of qualified staff with the technical competence needed to carry out specific examinations. 
The ISO 15189 is a worldwide International Standard, specifically designed for the accreditation of medical laboratories. The ISO 15189 accreditation is issued by the national accreditation body, a unique body recognized by the national government for the issuing of accreditation. Obligatory in some countries and voluntary in others, accreditation is based on comparative logics between homogeneous organizations, typical of benchmark systems. Its purpose is not to satisfy a fixed target but goals that vary continuously over time and space and hinge upon quality of professional performance (best practice). Since best practice is affected by contextual change, technological evolution, scientific advancements, and so forth, the aim of accreditation is to help the laboratory achieve continued improvement and compare its performance with the best possible performance, as determined by the state-of-the-art and/or the reference model. The maintenance of accreditation, a dynamic process, calls for continuous evaluation of excellence in the respective discipline.

\subsubsection{Quality Indicators}

Despite the availability of laboratory accreditation and compliance with mandatory and/or voluntary standards calling for the application of the best available criteria and procedures, and the harmonization of staff behavior, its effectiveness is closely related to continuous monitoring of all TTP steps and outcomes.

In recent years, laboratory professionals have developed and implemented a number of QIs focusing on the main critical TTP steps that are to be included in a coherent and well-integrated quality improvement system [36-41]. This has come about because of the need to reduce error rates, the difficulty in recognizing and isolating undesired events, and a willingness to meet ISO 15189 accreditation requirements.

An internal evaluation process and participation in an inter-laboratory comparison must be implemented in order to guarantee the effectiveness of QIs.
The internal evaluation process includes the definition of:

- A list of QIs focused on the critical activities.

- A document for each indicator describing its specifications (e.g., what must be measured, how to collect data, measurement limitations, acceptability limits of results, areas of application, responsibilities).

- A standard operating procedure describing all steps to be followed in order to guarantee effective use of QIs.

Moreover, participation in inter-laboratory comparison aims to evaluate the quality level achieved in comparison with other national or international laboratories. This calls for the use of common QIs and of criteria and procedures for data collection. A coordinator center is needed to perform data processing that complies with approved quality specifications and to provide each laboratory with a report describing the resulting performance evaluation pertaining to each QI.

The Working Group on "Laboratory Errors and Patient Safety" (WG-LEPS) of the International Federation of Clinical Chemistry and Laboratory Medicine (IFCC) has put in motion a project for the definition of a Model of Quality Indicators (MQI) along with data collection specifications, to be used in all laboratories regardless of logistics, technological level, and complexity. In two Consensus Conferences held in 2013 and 2016, experts on this topic discussed and ultimately approved the list of QIs, the procedure for data collection, the criteria for performance evaluation, and the information to be included in a periodic report concerning the analysis of data for participating laboratories. The progress of the project and its findings are reported in literature as well as through a dedicated website that is www.ifccmqi.com.

Currently the MQI includes 53 measurements to evaluate 26 indicators concerning key processes, support processes, and outcome measures. To facilitate implementation, a priority 
Fig. 24.2 Model of Quality Indicators proposed by WG-LEPS of IFCC
IFCC Working Group “Laboratory Errors and Patient Safety”

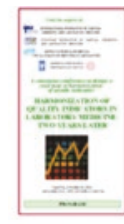

Model of Quality Indicators

26 Indicators

53 Measurements

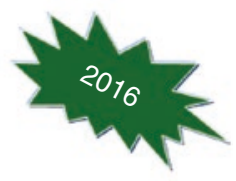

Indicators Measurements

Key Processes

Pre-analytical phase

Intra-analytical phase

Post-analytical phase

Support Processes

$\begin{array}{rr}20 & 43 \\ 11 & 25 \\ 5 & 6 \\ 4 & 12 \\ 3 & 5 \\ 3 & 5\end{array}$

Fig. 24.3 Model of Quality Indicators proposed by WG-LEPS of IFCC per priority index

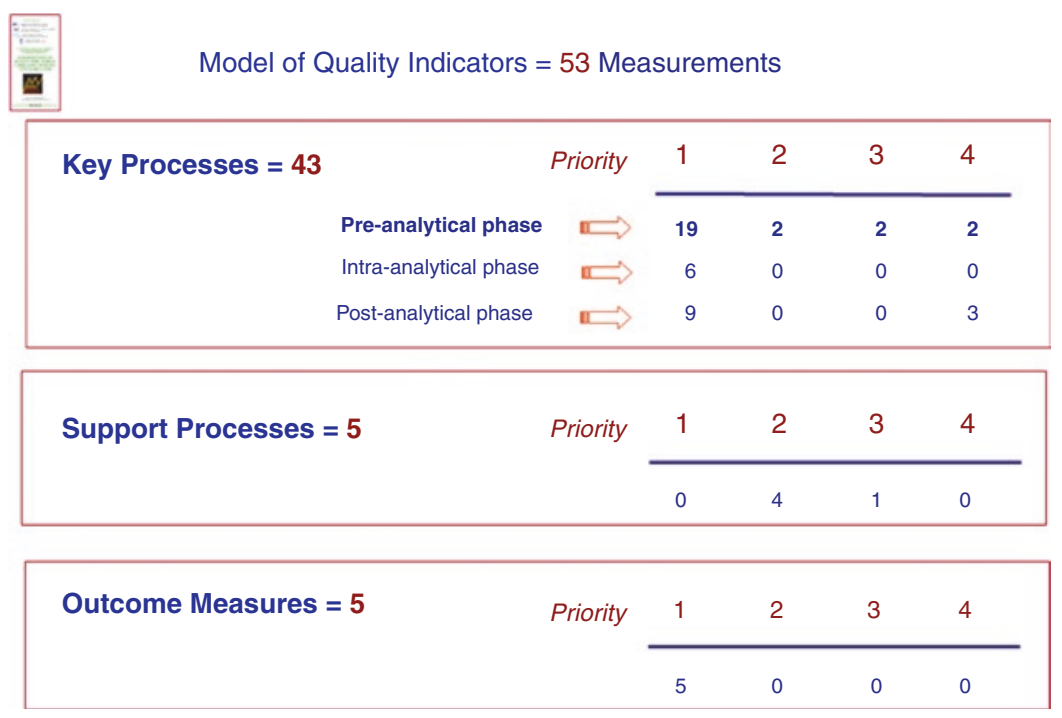

index has been assigned to each QI (from 1 to 4 , with 1 being the highest and 4 the lowest) based on the importance of the QI and the difficulty of data collection (Figs. 24.2 and 24.3).

However, sufficient effectiveness of the system cannot be achieved without the correct identification and analysis of the causes for error as well as the implementation of adequate corrective actions. In order to reduce error rates and improve laboratory performance, it is extremely important for laboratory professionals to periodically analyze the QI data and:
- Highlight declines in performance in comparison with previous data and with the state-ofthe-art (i.e., other laboratories).

- Identify any error-related causes of undesirable performance as well as any room for improvement.

- Implement preventative and/or corrective and/ or improvement actions.

- Evaluate any risk to patient safety.

QI data are an effective starting point for evaluating error probability in risk management pro- 
cedures. Some authors have reported on their experience applying Failure Mode and Effect Analysis (FMEA) to critical activities using QI data. The reported findings demonstrate that the use of QIs to monitor errors and implement risk management procedures reduces the error rate, maximizes performance quality, and improves patient safety and health system outcomes [42]. The promotion of continued improvement centers about the commitment of laboratory professionals to the management of QIs. The last few years have confirmed that the utility of QIs is closely linked to the recognition by all personnel involved of their importance and of the need to guarantee appropriate data collection as well as effective data analysis. To raise awareness in professionals, there has been a diffusion of consensually approved MQI and results that highlight achieved improvement, and professionals have been encouraged to assume responsibility. The continuous exchange of experiences among laboratory professional aim therefore to improve both the quality of the project and of laboratory services [41].

\subsubsection{Professional Competence: Education and Skill}

In order to make the role of laboratory medicine in the context of patient care more visible, laboratory professionals must accept that they are members of a multidisciplinary team. Greater visibility in the form of rounds, committees, consultation, demonstration of knowledge, and self-promotion will be judged chiefly in relation to the clinical value they bring. Laboratory professionals must develop new competencies to highlight the contribution of laboratory information to patient management. Even knowledge of less traditional areas is required; this will bring new insights and approaches from other disciplines. Moreover, laboratory professionals must realize that the level of recognition achieved will be profoundly affected by continuous advancements in areas such as computerization, technology, clinical decision tools, informative media, and artificial neural networks. Laboratory medicine is a con- tinuously evolving clinical discipline and emerging challenges require a revision and improvement of operational flows to enhance quality and safety in patients care. Laboratory professionals must maintain a high level of skills for achieving efficiency and effectiveness when delivering laboratory services [43]. To reduce diagnostic errors, the first-line intervention consists of education and training designed to improve knowledge and skills to guarantee relevant competency [23]. Given the data reported above, it might be necessary to act on graduate education and training by rescheduling national programs, enhancing the duration and contents of courses given in laboratory medicine. Currently, however, only postgraduate initiatives are underway. The EFLM published the fifth edition of a syllabus for laboratory medicine outlining requirements for the postgraduate skills, knowledge, and competency needed to direct laboratory medicine services. The syllabus would not replace existing postgraduate programs, the aim being to harmonize the common principles of education and training of professionals working Europe in order to guarantee high standards of quality and safety. This initiative paves the way for the free migration of professionals and patients across EU national borders. While the fourth version of the syllabus was built with the specialist's generic skills, knowledge, and competencies in mind, the fifth version details individual discipline requirements (such as those in clinical chemistry, immunology, hematology, blood transfusion, microbiology/ virology, genetics, and in vitro fertilization) and includes new analytical techniques and statistics. Laboratory organization, quality, safety, and clinical governance have also been included as fundamental aspects of training, thus enabling the specialist in Laboratory Medicine to operate as a clinical leader who can support and transform healthcare services [44]. Given the recent changes in the nature of laboratory service and its role in the healthcare process, the new generation of laboratory professionals and leaders are then called upon to incorporate specific technical skills into a broader vision of healthcare and of patients' needs. As shown in Table 24.1, the recently published "Manifesto for the future of 
laboratory medicine professionals" specifies the tasks of laboratory professionals in ten specific points. Clinical laboratory stewardship appears to be the new, shared strategy for guaranteeing patient safety while simultaneously maximizing efficacy and efficiency [1]. With this approach, quality and safety are held to be just as essential in daily laboratory practice as they are in clinical practice; yet, neither topic appears in national education and training programs.

The World Health Organization (WHO) has developed initiatives for professionals training in the field of patient safety, beginning with the publication of a "Multi-professional's patient safety curriculum guide" to provide assistance in the teaching of patient safety in universities and schools. This manual, originally published in 2011, has been translated into many different languages (i.e., Chinese, Czech, English, French, German, Indonesian, Italian, Japanese, Polish, and Spanish) [45]. In 2018, WHO organized the first international meeting on safety in healthcare addressed to students and residents in the medical field. More than 200 residents of different specialities and coming from 30 nations were divided into 18 working groups and enhanced the new clinical generation's perspective on healthcare safety through their participation. The survey administered at the end of the sessions highlighted that for $90 \%$ of respondents this was the first time attending an international meeting on patient safety, that $80 \%$ of residents had not been aware of the existence of the "WHO Multiprofessional Patient Safety Curriculum Guide" prior to attending, and that only $40 \%$ of the participants had already received training in the field of patient safety and clinical risk.

The above percentages show that in the areas of quality, safety, and risk management the training of young laboratory and clinical professionals is still linked to the sensitivity of leadership towards these issues. However, professionals cannot overlook this issue: they must all be aware that many laboratory errors stem from personnel-related factors, and that other factors can positively or negatively affect laboratory activities, factors such as the social environment, developments in technology, economic parameters, rules and regulations, and safety precautions [46-48].

\subsubsection{Risk Management Procedures}

Risk management, the systemic process designed to identify and manage the actual and potential risks associated with laboratory testing, is becoming an integral part of quality management systems and plays an important role in providing quality services [49].

Although ISO 15189 [36] and ISO 9001 [50] include risk management requirements, they do not specify the means to this end. Laboratory professionals generally choose risk assessment techniques recommended by ISO/TS 22367 [20] or suggested by CLSI EP18-A2 [51] while using differing approaches to define goals and identify risks.

Available reviews of risk management procedures in literature focus on different steps and activities. Some authors use FMEA and the Failure Reporting, Analysis and Corrective Action System (FRACAS) to estimate, respectively, potential and actual risks associated with operational (i.e., pre-analytical, analytical, and post-analytical) and strategic and support processes [52]. Other authors use the FMEA technique for specific examination procedures to reduce the occurrence of multiple failures recorded throughout the entire testing process, in particular risks concerning Factor $\mathrm{V}$ Leiden mutation or parathyroid and adrenocorticotropic hormones [53, 54], or risks pertaining to the preanalytical phase using QI data [42].

The use of QI data in risk management procedures is a valid mean for evaluating the probability of error occurrence. The Australasian QI program, Key Incident Monitoring and Management System (KIMMS), provides participants with the KIMMS risk matrix among other statistical tools in order to encourage laboratories to examine high-risk steps and analyze causes of error. For each QI, the system automatically multiplies together the frequency imputed by lab 
participants, the harm as defined in advance by a consensus of lab professionals, and the ability to detect errors [55]. Flegar-Meštrić et al. [42] also retrospectively studied data collected from 22 harmonized QIs of MQI launched by the IFCC WG-LEPS for risk analysis and error reduction in pre-analytical steps in an emergency department with a higher error rate [33].

\subsection{Clinical Cases}

In our laboratory, the IFCC-MQI reports provided by WG-LEPS - including the mean and sigma value of laboratory performances for each quality indicator - are analyzed annually to identify high-risk processes. In 2018, the most frequent errors were related to sample unsuitability (e.g., hemolyzed, clotted, or insufficient samples, and samples with inappropriate sampleanticoagulant volume ratio of $0.262 \%$ ); as a result, the blood collection phase was analyzed to identify the high-risk steps and procedures. Errors in blood collection can generate incorrect results, delays in the release of results, delays in diagnosis and treatment, the release of incomplete testing panels, and repeated blood collection resulting in a negative final outcome for the patient. Checks already in place to prevent procedural errors included standard operating procedures and a training course for blood collection, as well as a checklist serving as an auxiliary tool to guide phlebotomists. All procedural errors can be detected in the laboratory by means of serum indices. To analyze the blood collection phase and identify causes of errors, a selection was made of five wards (i.e., neonatal pathology, clinical medicine, hematology, clinical immunology and day-hospital, and general medicine) with different organizational structure and patient characteristics. The blood collection phase was mapped out in each ward and the risk index (RI) estimated. The highest risk index was found for neonatal pathology $(\mathrm{RI}=226)$, followed by hematology $(\mathrm{RI}=165)$, clinical medicine $(\mathrm{RI}=138)$, clinical immunology day-hospital $(\mathrm{RI}=107)$, and general medicine $(\mathrm{RI}=63)$. The risk analysis demonstrated that the major causes of error were partial knowledge of the standard operating procedures relating to blood sampling and the storage and sending of the sample (for example, due to insufficient user credentials to access an internal website containing standard operating procedures), insufficient and/or inadequate staff training, partial use of the blood collection checklist, incorrect storage of the sample, human factors (e.g., stress, fatigue, lack of sleep), and the patient's condition (e.g., fragile veins). In order to reduce the error rate during blood collection and minimize risk to patients, several corrective actions were implemented: divulgation of the existing standard operating procedures on blood collection and on sample storage and transportation to the laboratory; request-based authorization to access the documentation found in the internal website; a training course on blood collection addressed to all phlebotomists; and encouraging use of the checklist. Six months after the implementation of the corrective actions, the risk index was re-evaluated to verify effectiveness. We found risk had been reduced in all the studied wards, in particular hematology $(58 \%$ risk minimization; RI $=69$ ), followed by neonatal pathology $(32 \%$; RI $=153)$, clinical medicine $(30 \%$; RI $=98)$, clinical immunology dayhospital $(28 \%$; RI $=77)$, and general medicine $(19 \%$; RI = 51).

The detection, identification, and monitoring of errors through a set of harmonized, evidencebased, and patient-centered QIs have allowed a better understanding and management of the more critical stages of the processes. QIs incorporated in the laboratory quality management system proved to be effective tools for risk assessment and minimized the possibility of errors occurring, consequently guaranteeing patient safety.

\subsection{Recommendations}

The prevention of errors in healthcare is still a worldwide priority for ensuring patient safety. Data reported by the World Health Organization 
(WHO) website on the incidence and magnitude of errors in healthcare are discouraging: it has been estimated that approximately 43 million patient safety incidences occur every year, and as many as one in ten patients are harmed while receiving healthcare. Medical record reviews have demonstrated that $6-17 \%$ of all adverse events in hospital are due to diagnostic errors, which have therefore been listed among the ten factors affecting patient safety [56].

The IOM defined patient safety as "the prevention of harm to patients," considering it "indistinguishable from the delivery of quality healthcare," and defined quality of care as "the degree to which healthcare services for individuals and populations increase the likelihood of desired health outcomes and are consistent with current professional practices" [46, 57]. It is clear from these definitions that safety is an essential building block for high-quality performance and that it is strictly connected to the other dimensions of quality of care, such as patientcenteredness, effectiveness, timeliness, efficiency, and equity [58].

Quality assurance tools in laboratory medicine must be integrated within the everyday work of all laboratory professionals, who must shift from focusing on individual human errors to adopting a systematic approach.

\section{References}

1. Plebani M, Laposata M, Lippi G. A manifesto for the future of laboratory medicine professionals. Clin Chim Acta. 2019;489:49-52.

2. Plebani M. The future of laboratory medicine: navigating between technology and professionalism. Clin Chim Acta. 2019;49:816.

3. Plebani M. Towards a new paradigm in laboratory medicine: the five rights. Clin Chem Lab Med. 2016;54:1881-91.

4. Brush JE, Brophy JM. Sharing the process of diagnostic decision making. JAMA Intern Med. 2017;177:1245-6.

5. Schiff GD, Martin SA, Eidelman D, Volk I, Ruan $\mathrm{E}$, Cassel C, et al. Ten principles for more conservative, care-full diagnosis. Ann Intern Med. 2018;169(9):643-64.

6. Kalra J. Medical errors: impact on clinical laboratories and other critical areas. Clin Biochem. 2004;37:1052-62.
7. Lundberg GD. Acting on significant laboratory results. JAMA. 1981;245:1762-3.

8. Plebani M, Laposata M, Lundberg GD. The brain-tobrain loop concept for laboratory testing 40 years after its introduction. Am J Clin Pathol. 2011;136:829-33.

9. Plebani M. The CCLM contribution to improvements in quality and patient safety. Clin Chem Lab Med. 2013;51:39-46.

10. Dintzis SM, Stetsenko GY, Sitlani CM, Gronowski AM, Astion ML, Gallagher TH. Communicating pathology and laboratory errors: anatomic pathologists' and laboratory medical directors' attitudes and experiences. Am J Clin Pathol. 2011;135:760-5.

11. Himmel ME, Lam K, Fralick M. Hemodialysis in a healthy patient - a case of an erroneous laboratory result: a teachable moment [published erratum appears in JAMA Intern Med 2016;176:1037]. JAMA Intern Med. 2016;176:431-2.

12. Bellandi T, Albolino S, Tartaglia R, Filipponi F. Unintended transplantation of three organs from an HIV-positive donor: report of the analysis of an adverse event in a regional health care service in Italy. Transplant Proc. 2010;42:2187-9.

13. Frias JP, Lim CG, Ellison JM, Montandon CM. Review of adverse events associated with false glucose readings measured by GDH-PQQ-based glucose test strips in the presence of interfering sugars. Diabetes Care. 2010;33:728-9.

14. Ross JW, Boone DJ. Institute on Critical Issues in Health Laboratory Practice, vol. 173. Wilmington, DE: DuPont Press; 1989.

15. Plebani M, Carraro P. Mistakes in a stat laboratory: types and frequency. Clin Chem. 1997;43:1348-51.

16. Carraro P, Plebani M. Errors in a stat laboratory: types and frequencies 10 years later. Clin Chem. 2007;53:1338-42.

17. Plebani M, Lippi G. Improving diagnosis and reducing diagnostic errors: the next frontier of laboratory medicine. Clin Chem Lab Med. 2016;54:1117-8.

18. Laposata M, Dighe A. "Pre-pre" and "post-post" analytical error: high-incidence patient safety hazards involving the clinical laboratory. Clin Chem Lab Med. 2007;45:712-9.

19. Astion ML, Shojania KG, Hamill TR, Kim S, Ng VL. Classifying laboratory incident reports to identify problems that jeopardize patient safety. Am J Clin Pathol. 2003;120:18-26.

20. International Organization for Standardization (ISO). ISO Technical Report 22367:2008. Medical laboratories-reduction of error through risk management and continual improvement-complementary elements. Geneva: International Organization for Standardization; 2008.

21. Committee on Patient Safety and Health Information Technology, Institute of Medicine. Health IT and patient safety: building safer systems for better care. Washington, DC: National Academies Press; 2011.

22. Plebani M. Laboratory-associated and diagnostic errors: a neglected link. Diagnosis (Berl). 2014;1:89-94. 
23. World Health Organization. Diagnostic errors: technical series on safer primary care. Licence: CC BY-NC-SA 3.0 IGO. Geneva: World Health Organization; 2016.

24. Schiff GD, Hasan O, Kim S, Abrams R, Cosby K, Lambert BL, et al. Diagnostic error in medicine: analysis of 583 physician-reported errors. Arch Intern Med. 2009;169:1881-7.

25. Gandhi TK, Kachalia A, Thomas EJ, Puopolo AL, Yoon C, Brennan TA, Studdert DM. Missed and delayed diagnoses in the ambulatory setting: a study of closed malpractice claims. Ann Intern Med. 2006;145:488-96.

26. Kachalia A, Gandhi TK, Puopolo AL, Yoon C, Thomas EJ, Griffey R, et al. Missed and delayed diagnoses in the emergency department: a study of closed malpractice claims from 4 liability insurers. Ann Emerg Med. 2007;49:196-205.

27. Plebani M. Errors in clinical laboratories or errors in laboratory medicine? Clin Chem Lab Med. 2006;44:750-9.

28. Vogeser M, Seger C. Quality management in clinical application of mass spectrometry measurement systems. Clin Biochem. 2016;49:947-54.

29. Vogeser M, Seger C. Irregular analytical errors in diagnostic testing - a novel concept. Clin Chem Lab Med. 2018;56:386-96.

30. Ismail AA. When laboratory tests can mislead even when they appear plausible. Clin Med (Lond). 2017; 17:329-32.

31. Plebani M. Errors in laboratory medicine and patient safety: the road ahead. Clin Chem Lab Med. 2007;45:700-7.

32. Hofgärtner WT, Tait JF. Frequency of problems during clinical molecular-genetic testing. Am J Clin Pathol. 1999;112:14-21.

33. Sciacovelli L, Lippi G, Sumarac Z, West J, Garcia Del Pino Castro I, Furtado Vieira K, et al. Working Group "Laboratory Errors and Patient Safety" of International Federation of Clinical Chemistry and Laboratory Medicine (IFCC). Quality indicators in laboratory medicine: the status of the progress of IFCC Working Group "Laboratory Errors and Patient Safety" project. Clin Chem Lab Med. 2017;55:348-57.

34. Plebani M. The journey toward quality and patient safety in laboratory medicine continues. North Am J Med Sci. 2014;6:229-30.

35. Boone DJ. Assessing laboratory employee competence. Arch Pathol Lab Med. 2000;124:190-1.

36. International Organization for Standardization. ISO 15189:2012. Medical laboratories-Requirements for quality and competence. Geneva: International Organization for Standardization; 2012.

37. Wagar EA, Tamashiro L, Yasin B, et al. Patient safety in the clinical laboratory: a longitudinal analysis of specimen identification errors. Arch Pathol Lab Med. 2006;130:1662-8.

38. Lippi G, Blanckaert N, Bonini P, et al. Causes, consequences, detection, and prevention of identification errors in laboratory diagnostics. Clin Chem Lab Med. 2009;47:143-53.

39. Plebani M. Quality indicators to detect pre-analytical errors in laboratory testing. Clin Biochem Rev. 2012;33:85-8.

40. Plebani M, Sciacovelli L, Aita A, et al. Quality indicators to detect pre-analytical errors in laboratory testing. Clin Chim Acta. 2014;432:44-8.

41. Plebani M, Sciacovelli L, Marinova M, et al. Quality indicators in laboratory medicine: a fundamental tool for quality and patient safety. Clin Biochem. 2013;46:1170-4.

42. Flegar-Mestric Z, Perkov S, Radeljak A, Marijana M, Paro K, Prkacin I, Devcic-Jeras A. Risk analysis of the pre-analytical process based on quality indicators data. Clin Chem Lab Med. 2017;55:368-77.

43. McQueen MJ. Will physicians and scientists have any role in managing laboratory resources in the year 2002. Eur J Clin Chem Clin Biochem. 1996;34:867-71.

44. Jassam N, Lake J, Dabrowska M, Queralto J, Rizos D, Lichtinghagen R, et al. The European Federation of Clinical Chemistry and Laboratory Medicine syllabus for postgraduate education and training for Specialists in Laboratory Medicine: version 5-2018. Clin Chem Lab Med. 2018;56:1846-63.

45. World Health Organization. Multi-professional patient safety curriculum guide. Geneva: World Health Organization; 2011.

46. Institute of Medicine (US) Committee on Quality of Health Care in America. In: Kohn LT, Corrigan JM, Donaldson MS, editors. To err is human: building a safer health system. Washington, DC: National Academies Press; 2000.

47. Plebani M. System-related and cognitive errors in laboratory medicine. Diagnosis (Berl). 2018;5:191-6.

48. Laposata M. Obtaining a correct diagnosis rapidly in the United States is associated with many barriers not present in other countries. Am J Clin Pathol. 2018;149:458-60.

49. Scally G, Donaldson LJ. The NHS's 50 anniversary. Clinical governance and the drive for quality improvement in the new NHS in England. BMJ. 1998;317:61-5.

50. International Organization for Standardization (ISO). ISO 9001:2015. Quality management system. Geneva: International Organization for Standardization (ISO); 2015.

51. Clinical and Laboratory Standards Institute (CLSI). CLSI EP18-A2. Risk management techniques to identify and control error sources. Approved Guideline. 2nd ed. USA; 2009.

52. Lao EG, Garcìa AS, Figuerola MB, Moreno E, Paraire $\mathrm{AH}$. Errors of clinical laboratories and its impact on patient safety. Open J Soc Sci. 2017;5:243-53.

53. Serafini A, Troiano G, Franceschini E, Calzoni P, Nante N, Scapellato C. Use of a systematic risk analysis method (FMECA) to improve quality in a clinical laboratory procedure. Ann Ig. 2016;28:288-95. 
54. Magnezi R, Hemi A, Hemi R. Using the failure mode and effects analysis model to improve parathyroid hormone and adrenocorticotropic hormone testing. Risk Manag Healthc Policy. 2016;9:271-4.

55. Badrick T, Gay S, Mackay M, Sikaris K. The key incident monitoring and management system - history and role in quality improvement. Clin Chem Lab Med. 2018;56:264-72.

56. World Health Organization: WHO. http://www.who. int/features/factfiles/patient_safety/en/.
57. Institute of Medicine. Medicare: a strategy for quality assurance: executive summary IOM Committee to design a strategy for quality review and assurance in Medicare. Washington, DC: National Academy Press; 1990.

58. National Patient Safety Foundation. Free from harm: accelerating patient safety improvement fifteen years after. To err is human. National Patient Safety Foundation: Boston, MA; 2015.

Open Access This chapter is licensed under the terms of the Creative Commons Attribution 4.0 International License (http://creativecommons.org/licenses/by/4.0/), which permits use, sharing, adaptation, distribution and reproduction in any medium or format, as long as you give appropriate credit to the original author(s) and the source, provide a link to the Creative Commons license and indicate if changes were made.

The images or other third party material in this chapter are included in the chapter's Creative Commons license, unless indicated otherwise in a credit line to the material. If material is not included in the chapter's Creative Commons license and your intended use is not permitted by statutory regulation or exceeds the permitted use, you will need to obtain permission directly from the copyright holder. 